\title{
Nocturnal Heart Rate Variability Spectrum Characterization in Preschool Children with Asthmatic Symptoms
}

\author{
Javier Milagro, Eduardo Gil, Jesús Lázaro, Ville-Pekka Seppä, L. Pekka Malmberg, Anna S. Pelkonen, \\ Anne Kotaniemi-Syrjänen, Mika J. Mäkelä, Jari Viik and Raquel Bailón
}

\begin{abstract}
Asthma is a chronic lung disease that usually develops during childhood. Despite that symptoms can almost be controlled with medication, early diagnosis is desirable in order to reduce permanent airway obstruction risk. It has been suggested that abnormal parasympathetic nervous system (PSNS) activity might be closely related with the pathogenesis of asthma, and that this PSNS activity could be reflected in cardiac vagal control. In this work, an index to characterize the spectral distribution of the high frequency (HF) component of heart rate variability (HRV), named peakness $(\wp)$, is proposed. Three different implementations of $\wp$, based on electrocardiogram (ECG) recordings, impedance pneumography (IP) recordings and a combination of both, were employed in the characterization of a group of preschool children classified attending to their risk of developing asthma. Peakier components were observed in the HF band of those children classified as high-risk $(p<0.005)$, who also presented reduced sympathvoagal balance. Results suggest that high-risk of developing asthma might be related with a lack of adaptability of PSNS.
\end{abstract}

Index Terms-heart rate variability, asthma, children, peakness, parasympathetic nervous system.

\section{ACRONYMS}

$\begin{array}{ll}\text { ANS } & \text { Autonomic Nervous System } \\ \text { BMI } & \text { Body Mass Index } \\ \text { COPD } & \text { Chronic Obstructive Pulmonary Disease }\end{array}$

This work was supported by grant BES-2015-073694 and project TIN201453567-R from Ministerio de Economía y Competitividad. Also by Goverment of Aragón and European Social Fund (EU) through BSICoS group (T96), by CIBER in Bioengineering, Biomaterials \& Nanomedicine (CIBER-BBN) through Instituto de Salud Carlos III, by Helsinki University Research Grants, by Sigrid Juselius Foundation, by Tampere Tuberculosis Foundation and by Finnish Medical Foundation. This project has received funding from the European Unions Framework Programme for Research and Innovation Horizon 2020 (2014-2020) under the Marie Skłodowska-Curie Grant Agreement No. 745755. The computation was performed by the ICTS NANBIOSIS, specifically by the High Performance Computing Unit of CIBER-BBN at University of Zaragoza. Asterisk indicates corresponding author.

J. Milagro*, E. Gil and R. Bailón are with the Biomedical Signal Interpretation and Computational Simulation (BSICoS) group at the Aragón Institute of Engineering Research (I3A), IIS Aragón, University of Zaragoza, Zaragoza, and CIBER de Bioingeniería, Biomateriales y Nanomedicina (CIBER-BBN), Madrid, Spain, (e-mail: milagro@unizar.es).

J. Lázaro is with Department of Electrical Engineering (ESAT), STADIUS Centre for Dynamical Systems, Signal Processing and Data Analytics, KU Leuven, and IMEC, Leuven, Belgium.

J. Viik and V-P. Seppä are with BioMediTech Institute and Faculty of Biomedical Sciences and Engineering, Tampere University of Technology, Tampere, Finland.

L. P. Malmberg, A. S. Pelkonen, A. Kotaniemi-Syrjänen and M. J. Mäkelä are with Skin and Allergy Hospital, University of Helsinki and Helsinki University Hospital, Helsinki, Finland.

Manuscript received Month XX, 20XX; revised Month XX, 20XX.

$\begin{array}{ll}\text { ECG } & \text { Electrocardiogram } \\ \text { EDR } & \text { ECG-Derived Respiration } \\ \text { HF } & \text { High Frequency } \\ \text { HiR } & \text { High Risk } \\ \text { HR } & \text { Heart Rate } \\ \text { HRV } & \text { Heart Rate Variability } \\ \text { ICS } & \text { Inhaled Corticosteroids } \\ \text { IP } & \text { Impedance Pneumography } \\ \text { IQR } & \text { Inter-Quartile Range } \\ \text { LF } & \text { Low Frequency } \\ \text { LoR } & \text { Low Risk } \\ \text { mAPI } & \text { Modified Asthma Predictive Index } \\ \text { NN } & \text { Normal-to-Normal } \\ \text { OSAS } & \text { Obstructive Sleep Apnea Syndrome } \\ \text { pNN50 } & \text { Percentaje of successive Normal-to-Normal } \\ & \text { differences greater than 50 ms } \\ \text { PSD } & \text { Power Spectral Distribution } \\ \text { PSNS } & \text { Parasympathetic Nervous System } \\ \text { SDNN } & \text { Standard Deviation of Normal-to-Normal intervals } \\ \text { SDSD } & \text { Standard Deviation of Successive Differences } \\ \text { RMSSD } & \text { Root Mean Square of Successive Differences } \\ \text { RSA } & \text { Respiratory Sinus Arrhythmia } \\ \text { SNR } & \text { Signal-to-Noise Ratio } \\ \text { SPT } & \text { Skin Prick Test }\end{array}$

\section{INTRODUCTION}

A STHMA is a chronic inflammatory disease that narrows the airways [1], thus making breathing difficult. Although it affects people of any age [2] it is prone to start in childhood [3], and an increase in the number of asthmatic children during the last decades [2] has risen asthma as the most common chronic disease of childhood in the United States [4]. Asthma is characterized by a series of symptoms that include bronchial hyper-responsiveness, spasmodic contraction of the bronchioles or increase in mucus segregation among others, which cause difficulty for expiring the air from the lungs [5] and whose continuous presence may lead to the permanent remodeling of the airways [6].

Most of these symptoms are usually reversible with medication but in some cases they can turn severe [2], and hence accurate and early diagnosis is important in order to provide an appropriate treatment and follow-up of the patients, as early intervention seems to prevent permanent airway obstruction 
TABLE I

CHARACTERISTICS OF THE CHILDREN IN THE DATA BASE. (WHILE CONTINUOUS VARIABLES ARE EXPRESSED AS MEDIAN (MIN-MAX), INTEGER VARIABles ARE DisPlayed AS N (\%). BMI: Body MASS IndeX, SPT: SKIN PRICK TEST.)

\begin{tabular}{l|llll} 
& LoR & HiR & ICS & Total \\
\hline $\mathbf{n}$ & 14 & 13 & 7 & 34 \\
Sex (male) & $6(43 \%)$ & $5(39 \%)$ & $4(57 \%)$ & $15(44 \%)$ \\
Age (years) & $5.0(3.4-6.6)$ & $4.6(3.4-6.8)$ & $5.1(3.8-6.7)$ & $4.9(3.4-6.8)$ \\
Height (cm) & $111(96-121)$ & $110(94-126)$ & $108(104-127)$ & $109(94-127)$ \\
Birth weight (kg) & $3.6(2.1-5.2)$ & $3.6(2.8-4.6)$ & $3.5(3.0-4.1)$ & $3.5(2.1-5.2)$ \\
BMI & $16.5(13.5-18.3)$ & $15.9(14.8-18.0)$ & $16.1(14.5-18.0)$ & $16.1(13.5-18.3)$ \\
Wheeze & $5(36 \%)$ & $13(100 \%)$ & $7(100 \%)$ & $26(77 \%)$ \\
Allergic rhinitis & $2(14 \%)$ & $3(23 \%)$ & $2(29 \%)$ & $7(21 \%)$ \\
Atopic dermatitis & $7(50 \%)$ & $7(54 \%)$ & $3(43 \%)$ & $17(50 \%)$ \\
SPT positivity & $3(21 \%)$ & $9(69 \%)$ & $5(71 \%)$ & $17(50 \%)$ \\
Parental asthma & $4(28 \%)$ & $6(46 \%)$ & $4(57 \%)$ & $14(41 \%)$ \\
\hline
\end{tabular}

[7]. Despite the fact that asthma usually develops during childhood, there is not a feasible means to early diagnose it [8]. The assessment of asthma in young children is mainly based on clinical history [9]-[11], which is retrospective in nature and can be incomplete in some cases. Also classical expiratory flow measurements that are widely used in adults, such as forced expiratory volume in one second and forced vital capacity, are strongly effort dependent and therefore usually not reliable in young children with limited cooperation [8]. In this way, it would be interesting to dispose of noninvasive methods that could be used for aiding in the diagnosis of asthma without interfering in children normal activity.

Pathogenesis of asthma has been related with an abnormal autonomic nervous system (ANS) function [12]-[14], particularly with its parasympathetic branch, as parasympathetic nervous system (PSNS) is involved in the control of bronchomotor tone [15] and bronchoconstriction mechanisms [16], [17]. As high frequency (HF) component of heart rate variability (HRV) spectrum mainly reflects PSNS activity [18], HRV analysis has been used to assess increased vagal tone in asthmatic subjects [13], [14]. This, together with the absence of sympathetic innervation of airway smooth muscle [16], [17] point to PSNS activity as an important underlying factor of the altered bronchomotor tone in asthmatics. In this way, parasympathetic bronchial control in asthma has been previously studied in adults and children through HRV analysis [13], [14], but to the authors knowledge no preschool children HRV studies have been performed.

Respiratory modulation of HF component of HRV spectrum varies over time. This modulation depends on breathing parameters and might be also related with the configuration of the whole tracheobronchial tree (macro-configuration) in a given instant, which in turn depends on each airway configuration (micro-configuration). Under the assumption of independent airways, Que et al. [19] suggested that probability of a simultaneous narrowing of all the airways tends to zero. However, increased bronchomotor tone in asthma produces a decreased airway caliber, which might lead to an increase of this probability. In this way, Que et al. hypothesized that asthma could be regarded as a state in which improbable airway micro-configuration distribution are more likely to occur, thus leading to a lack of adaptability. As the underlaying bronchoconstriction mechanisms are thought to be mainly driven by PSNS [16], [17], this lack of adaptability could be reflected in or caused by changes in PSNS activity behavior.

Hence, PSNS activity has been presented to be different in asthmatic children than in controls and these differences can be assessed through HRV analysis. Under the hypothesis that abnormal PSNS behavior in asthma may be reflected in changes in the spectral distribution of HF component of HRV spectrum, in this work we aim to characterice HRV spectra in preschool children that have been classified attending to their risk of developing asthma.

\section{MAterials AND Methods}

\section{A. Study population}

The data used in this work consists on electrocardiogram (ECG) holter and impedance pneumography (IP) recordings of 44 children who were referred to the Pediatric Allergy Unit of Helsinki University Hospital due to persistent or recurrent lower respiratory tract symptoms, such as wheezing (a whistling sound when expiring air from the lungs), shortness of breath or coughing. From this 44 recordings, 10 were discarded due to electrode detachment, patient turning off the device or forgetting to turn on the device. The recording devices and the ECG and IP acquisition were custom designed at Tampere University of Technology (Tampere, Finland) [20], and signals were acquired with a sampling frequency of $256 \mathrm{~Hz}$. The mean length of the recordings is about 14 hours ( \pm 3.5 hours).

Patients were classified into three groups according to their modified asthma predictive index (mAPI) [21]. Children with a positive mAPI were classified as a high risk (HiR) group for developing persistent asthma, whereas children with negative mAPI were classified as low risk (LoR). Furthermore, another group was formed with children with a confirmed history of wheeze but that were under inhaled corticosteroids (ICS) treatment at the time of the recording. In the case of $\mathrm{HiR}$ and 
LoR groups, none of the subjects was under regular asthma treatment, nor were they supplied bronchodilators during the recordings. Table I contains a summary of patient information. Data acquisition was approved by an institutional pediatric ethics review board and informed written consent was received from guardians of all patients. Also informed written parental consent was received before data acquisition.

This data was collected for the study performed by Seppä et al. [22], hence additional information can be found there.

\section{B. Preprocessing}

Signals were only analyzed during night due to several reasons. Cardiac vagal tone is increased at night [23], with a higher effect over bronchomotor tone in the case of asthmatic subjects [24]. Moreover, children activity during day time is usually higher than in the case of adults (and also unknown in the analyzed database), compromising the analysis and interpretation of the results in this period.

Analysis period was set between 23:00 and 05:00 based on the observation of a reduction in mean heart rate (HR) at this time interval, indicating a resting/sleeping state of the patients.

In a preprocessing step, ECG holter signals were resampled to $1000 \mathrm{~Hz}$ using cubic splines interpolation in order to reduce the impact of ECG sampling frequency on HRV analysis [25]. ECG baseline was extracted with a $3^{\text {rd }}$ order Butterworth low-pass filter with $0.5 \mathrm{~Hz}$ cut-off frequency to be further subtracted from the interpolated ECG. Detection of QRScomplexes in order to generate RR interval (time distance between consecutive QRS-complexes) series was performed with the wavelet-based approach described by Martínez et al. [26].

Visual analysis of the signals revealed the existence of some very noisy ECG segments, probably due to movement (movement artifacts). In order to exclude from the analysis those bad signal-quality ECG segments, beat-to-beat signal to noise ratio (SNR) was calculated from the ECG signals as described in Bailón et al. [27]. This beat-to-beat SNR was computed as:

$$
\hat{x}_{\mathrm{SNR} i}=\frac{A_{i}}{1+P_{N_{i}}}
$$

where $\hat{x}_{\mathrm{SNR}_{i}}, A_{i}$ and $P_{N_{i}}$ are the SNR, peak-to-peak amplitude and high-frequency noise level of beat $i$, respectively. $A_{i}$ was obtained as the difference between the maximum and the minimum value of the QRS-complex corresponding to beat $i$, whereas $P_{N_{i}}$ was calculated as:

$$
P_{N_{i}}=\sqrt{\frac{1}{t_{2}^{i}-t_{1}^{i}} \int_{t_{1}^{i}}^{t_{2}^{i}} x^{2}(t) \mathrm{d} t}
$$

where $x(t)$ is a high-pass filtered version of the ECG signal ( $2^{\text {nd }}$ order, $20 \mathrm{~Hz}$ cut-off frequency Butterworth filter) and $t_{1}^{i}$ is set $150 \mathrm{~ms}$ after the QRS-complex corresponding to beat $i$, whereas $t_{2}^{i}$ is dependent of the instantaneous $\mathrm{HR}$ and is calculated as $t_{1}^{i}+\mathrm{RR}_{i} / 2$, being $\mathrm{RR}_{i}$ the $\mathrm{RR}$ interval (in ms) between beat $i$ and beat $i+1$. Threshold for assuming
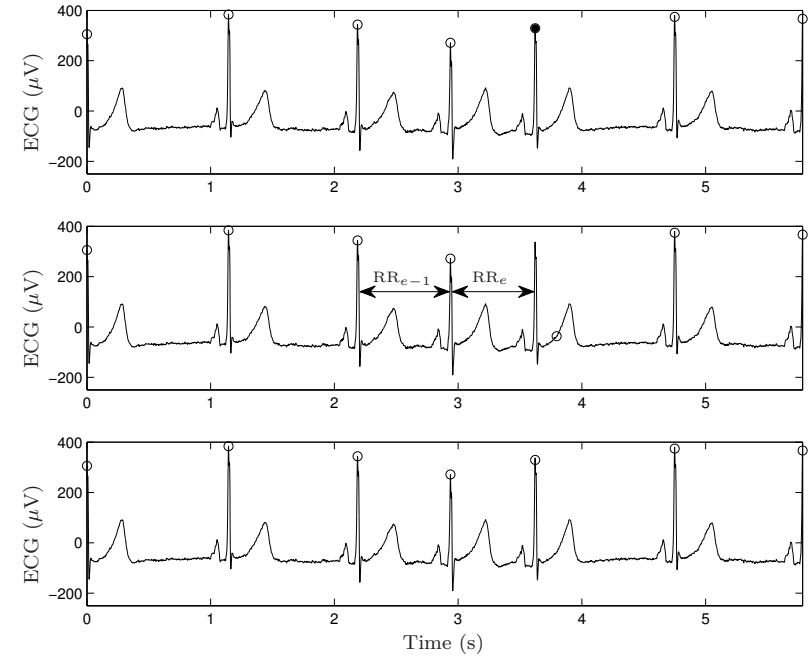

Fig. 1. In the upper panel, an ECG segment showing a RSA episode is displayed. After QRS-complex detection (empty circles), the method proposed by Mateo and Laguna in [28] labeled the beat marked with a filled circle as an ectopic. In the middle panel, the position of the beat labeled as ectopic has been incorrectly modified. In order to solve that, $\mathbf{R R}_{e}$ and $\mathbf{R R}_{e-1}$ are compared. As the condition $1.15 \cdot \mathrm{RR}_{e} \geq \mathrm{RR}_{e-1}$ is satisfied, the event is considered to be a RSA episode, and the correction is undone. The resulting detection is displayed in the lower panel.

acceptable SNR was established at $20 \mathrm{~dB}$ below the median SNR of the night period of the recording.

For ectopic beats detection, first, the method proposed by Mateo and Laguna [28], which imposes an upper threshold on the degree of variation of instantanous heart rate, was employed. However, since respiratory sinus arrhythmia (RSA) is stronger in children [29], some RSA episodes were considered as ectopic beats. So the identified ectopic beats were revised according to the following criteria: when a beat was labeled as ectopic the RR interval between it and the previous beat $\left(\mathrm{RR}_{e}\right)$ was compared with the previous $\mathrm{RR}$ interval $\left(\mathrm{RR}_{e-1}\right)$. If the condition $1.15 \cdot \mathrm{RR}_{e} \geq \mathrm{RR}_{e-1}$ (adopted from Tsipouras et al. [30]) was satisfied, the event was considered as a RSA episode instead of as an ectopic beat occurrence. An example of this process is shown in Fig. 1.

On the other hand, IP signals preprocessing consisted in a $3^{\text {rd }}$ order Butterworth band-pass filtering, with cut-off frequencies of 0.05 and $0.5 \mathrm{~Hz}$, in order to eliminate the baseline and discard all those components that are not expected to be respiration-related.

Both ECG and IP signals were later split in five-minute segments overlapped four minutes, so that a five-minute segment was obtained for each minute of the recording. Those segments whose ECG SNR decayed under the threshold were discarded.

\section{Respiratory frequency estimation}

Respiratory frequency was estimated within each fiveminute block by two different approaches. In the first one, the respiratory frequency was extracted directly from the power spectral density (PSD) of the IP signal as proposed by Bailón et al. [27], being referred to as $\mathrm{F}_{\mathrm{IP}}$. The PSD of the IP signal was calculated by Welch's method (50-second windows with 
$50 \%$ overlap). Also, respiratory frequency was estimated from the QRS slopes and R-wave angle ECG-derived respiration (EDR) approach proposed by Lázaro et al. [31]. The previous method (Bailón et al. [27]) was applied to the resulting EDR afterwards. The resulting frequency is referred to as $\mathrm{F}_{\mathrm{EDR}}$.

\section{HRV indexes}

In this work both time and frequency domain analyses were considered. In time domain analysis mean normal-tonormal interval (NN), SDNN, SDSD, RMSSD and pNN50 [18] were obtained. In the case of the frequency domain analysis, RR interval series were not directly transformed to the frequency domain, but the modulating signal that is thought to carry ANS information was first estimated according to the time-varying integral pulse frequency modulation (TVIPFM) model presented by Bailón et al. in [32] (in the following calculations, subindex $j$ represents the selected segment of a five minute block, from which also time domain measurements were obtained). First, instantaneous $\mathrm{HR}, d_{\mathrm{HR}_{j}}(n)$, was obtained from RR interval series and re-sampled at 4 Hz. Beat time occurrences of those beats labeled as ectopics were corrected with cubic spline interpolation (in average only $0.62 \%$ of the beats were ectopics, being them distributed in time). Afterwards, a time-varying mean $\mathrm{HR}, d_{\mathrm{HRM}_{j}}(n)$, was obtained by applying a $0.03 \mathrm{~Hz}$ low-pass filter to $d_{\mathrm{HR}_{j}}(n)$ and the HRV signal, $d_{\mathrm{HRV}_{j}}(n)$, was obtained as $d_{\mathrm{HRV}_{j}}(n)=$ $d_{\mathrm{HR}_{j}}(n)-d_{\mathrm{HRM}_{j}}(n)$. Finally, the modulating signal, $m_{j}(n)$, was calculated as $m_{j}(n)=d_{\mathrm{HRV}_{j}}(n) / \bar{d}_{\mathrm{HRM}_{j}}(n)$, where $\bar{d}_{\mathrm{HRM}_{j}}(n)$ is the mean of $d_{\mathrm{HRM}_{j}}(n)$ calculated over the $j$-th segment.

From this modulating signal, $m_{j}(n)$, HRV spectrum, $\hat{S}_{\mathrm{HRV}}(F)$, was estimated through Welch's periodogram (50second window, $50 \%$ overlap), and classical frequency indexes were calculated: low frequency (LF) and HF power $\left(\mathrm{P}_{\mathrm{LF}}\right.$ and $\mathrm{P}_{\mathrm{HF}}$ respectively), total power (TP), $\mathrm{P}_{\mathrm{LF}}$ to $\mathrm{P}_{\mathrm{HF}}$ ratio $\left(\mathrm{R}_{\mathrm{LF} / \mathrm{HF}}\right)$ and normalized LF power $\left(\mathrm{P}_{\mathrm{LFn}}\right)$ [18]. In this work, classical LF band in the range [0.04-0.15] Hz was considered [18]. On the other hand, HF band was defined as a $0.15 \mathrm{~Hz}$ window centered in the $\mathrm{F}_{\text {IP }}$ of the considered segment (from now, HF band refers to the modified band, whereas classical $\mathrm{HF}$ band refers to the [0.15-0.4] $\mathrm{Hz}$ band). The reason to modify it was that, in some cases, $\mathrm{F}_{\mathrm{IP}}$ was near $0.4 \mathrm{~Hz}$, so part of the power around it spread along frequencies larger than $0.4 \mathrm{~Hz}$. Also, there were some cases where frequency components with uncertain origin did appear around $0.15 \mathrm{~Hz}$ (mid-frequency band, described by Goren et al. [33]), so a bias would de introduced in the HF power calculation if considering them. It was checked that there were not cases where the lower bound of the resulting HF band was lower than $0.15 \mathrm{~Hz}$. An example showing both limitations is displayed in Fig. 2.

Minute by minute evolution of all the cited parameters was obtained from five-minute segments.

\section{E. Peakness}

An index for evaluating the distribution of the HF band of HRV spectrum is presented. Essentially, it is based on measuring how the most powerful frequency components of HF band are concentrated around the respiratory frequency. In

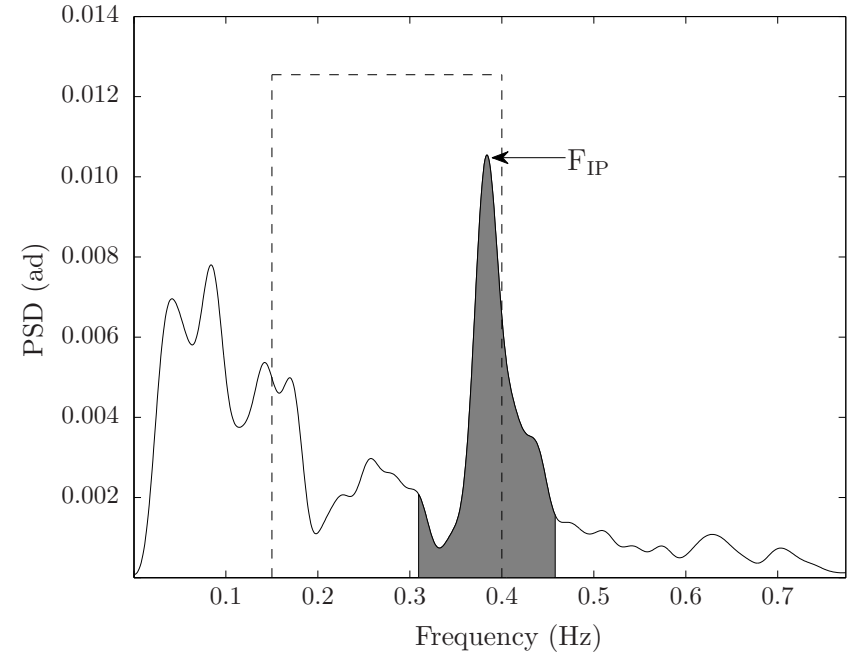

Fig. 2. PSD showing the $0.15 \mathrm{~Hz} \mathrm{HF}$ band (gray) centered in $\mathrm{F}_{\text {IP. The }}$ classical HF band of [0.15-0.4] Hz is shown with a dashed rectangle. It can be noticed that HF power would not be well estimated if classical HF band was considered: in the lower boundary we can observe spectral leakage from LF band, whereas in the higher boundary a leakage from HF outside the band due to the fact that the maximum is close to $0.4 \mathrm{~Hz}$ can be found. PSD, as obtained from the modulating signal $m(n)$ (see text for details), is adimensional (ad).

this way, the power concentrated in a narrow band around the respiratory frequency estimation was calculated as:

$$
\mathrm{P}_{\Delta}=\int_{\max \left(\mathrm{F}_{\mathrm{IP}}-\Delta f / 2,0.15\right)}^{\min \left(\mathrm{F}_{\mathrm{IP}}+\Delta f / 2, \overline{\mathrm{HR}} / 2\right)} \hat{S}_{\mathrm{HRV}}(F) \mathrm{d} F
$$

where $\Delta f$ is twice the resolution of Hamming window, $\Delta f=2.6 \frac{F_{s}}{N}$, being $F_{s}$ the sampling frequency and $N$ the number of samples of the window $(50 \mathrm{seg} \times 4$ samples/seg $=200$ samples). The limits of $[0.15-\overline{\mathrm{HR}} / 2] \mathrm{Hz}$ are imposed in order to ensure that no power from the LF band or from components with uncertain origin are considered $(\overline{\mathrm{HR}} / 2 \mathrm{~Hz}$ is the highest frequency we can represent in HRV spectrum, as HR is the intrinsic sampling frequency of HRV [34]).

The proposed index, named peakness $(\wp)$, is then defined as:

$$
\wp_{\mathrm{HRV}}^{\mathrm{IP}}=\frac{\int_{\max \left(\mathrm{F}_{\mathrm{IP}}-\Delta f / 2,0.15\right)}^{\min \left(\mathrm{F}_{\mathrm{IP}}+\Delta f / 2, \overline{\mathrm{HR}} / 2\right)} \hat{S}_{\mathrm{HRV}}(F) \mathrm{d} F}{\int_{\max \left(\mathrm{F}_{\mathrm{IP}}-\Delta F / 2,0.15\right)}^{\min \left(\mathrm{F}_{\mathrm{IP}}+\Delta F / 2, \overline{\mathrm{HR}} / 2\right)} \hat{S}_{\mathrm{HRV}}(F) \mathrm{d} F}=\frac{\mathrm{P}_{\Delta}}{\mathrm{P}_{\mathrm{HF}}}
$$

where the subindex HRV and the superindex IP indicate that $\wp_{\mathrm{HRV}}^{\mathrm{IP}}$ is calculated from the HRV spectrum and using $\mathrm{F}_{\mathrm{IP}}$ as the estimation of the respiratory frequency, and $\Delta F$ is a 0.15 $\mathrm{Hz}$ band centered in the estimated respiratory frequency. As seen in eq. (4), the value of $\wp_{\mathrm{HRV}}^{\mathrm{IP}}$ will be higher as the power of the HF band of the spectrum is more concentrated around a single frequency (see Fig. 3), being $\wp_{\mathrm{HRV}}^{\mathrm{IP}}=1$ in the case of a pure sinusoid.

In order to assess if IP signal can be excluded from the analysis, thus only considering ECG, another approach was proposed. In this case the respiratory frequency estimation 
used was the one obtained from the EDR. Hence, the new definition of $\wp$ is:

$$
\wp_{\mathrm{HRV}}^{\mathrm{EDR}}=\frac{\int_{\max \left(\mathrm{F}_{\mathrm{EDR}}-\Delta f / 2,0.15\right)}^{\min \left(\mathrm{F}_{\mathrm{EDR}}+\Delta f / 2, \overline{\mathrm{HR}} / 2\right)} \hat{S}_{\mathrm{HRV}}(F) \mathrm{d} F}{\int_{\max \left(\mathrm{F}_{\mathrm{EDR}}-\Delta F / 2,0.15\right)}^{\min \left(\mathrm{F}_{\mathrm{EDR}}+\Delta F / 2, \overline{\mathrm{HR}} / 2\right)} \hat{S}_{\mathrm{HRV}}(F) \mathrm{d} F}
$$

where the subindex HRV and the superindex EDR indicate that $\wp_{\mathrm{HRV}}^{\mathrm{EDR}}$ is calculated from the HRV spectrum and using $\mathrm{F}_{\mathrm{EDR}}$ as an estimation of the respiratory frequency.

Finally, the complementary case of using only the IP signal was considered. Hence, $\wp$ was derived directly from the PSD of the IP signals as:

$$
\wp_{\mathrm{IP}}^{\mathrm{IP}}=\frac{\int_{\max \left(\mathrm{F}_{\mathrm{IP}}-\Delta f / 2,0.15\right)}^{\min \left(\mathrm{F}_{\mathrm{IP}}+\Delta f / 2, \overline{\mathrm{HR}} / 2\right)} \hat{S}_{\mathrm{IP}}(F) \mathrm{d} F}{\int_{\max \left(\mathrm{F}_{\mathrm{IP}}-\Delta F / 2,0.15\right)}^{\min \left(\mathrm{F}_{\mathrm{IP}}+\Delta F / 2, \overline{\mathrm{HR}} / 2\right)} \hat{S}_{\mathrm{IP}}(F) \mathrm{d} F}
$$

where $\hat{S}_{\mathrm{IP}}(F)$ is the PSD of the IP signal and the subindex IP and the superindex IP indicate that $\wp_{\mathrm{IP}}^{\mathrm{IP}}$ is calculated from

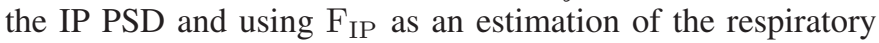
frequency.

\section{F. Statistical Methods}

Subject characterization was performed by calculating the mean and median of the minute by minute evolution for every possible combination of two consecutive hours in order to cover at least one complete sleep cycle [35]. Also inter-quartile range (IQR) was calculated. For the three study groups, a Kolmogorov-Smirnov test was applied for assessing normality of the data. Afterwards, a two-sided Wilcoxon rank-sum test was applied when needed to the described mean in order to assess differences between groups. Also Spearman correlation coefficient $(\rho)$ and Bland-Altman plot [36] were calculated for assessing the relationship between parameters. Finally, the mean absolute error in the estimation of $\mathrm{F}_{\mathrm{EDR}}$ was computed.

\section{RESULTS}

The highest number of significant differences between groups was found for the two-hour period going from 2 a.m. to 4 a.m. with similar results for the mean and median of all the parameters, so only mean is considered in the further discussion for simplification. Results obtained for this period are displayed in Table II, where it can be noticed that the three $\wp, \mathrm{R}_{\mathrm{LF} / \mathrm{HF}}$ and $\mathrm{P}_{\mathrm{LFn}}$ presented statistically significant differences between LoR and HiR. On the other hand, mean $\mathrm{P}_{\mathrm{HF}}, \mathrm{R}_{\mathrm{LF} / \mathrm{HF}}, \mathrm{P}_{\mathrm{LFn}}, \wp_{\mathrm{HRV}}^{\mathrm{IP}}$ and $\wp_{\mathrm{HRV}}^{\mathrm{EDR}}$ were able to distinguish between LoR and ICS, whereas only $R_{\mathrm{LF} / \mathrm{HF}}$ and $\mathrm{P}_{\mathrm{LFn}}$ presented significant differences between HiR and ICS.

In order to check the accuracy of the respiratory frequency estimation obtained from the EDR method, the mean absolute error between $F_{I P}$ and $F_{E D R}$ was calculated, being it $0.0038 \pm 0.0044 \mathrm{~Hz}$.
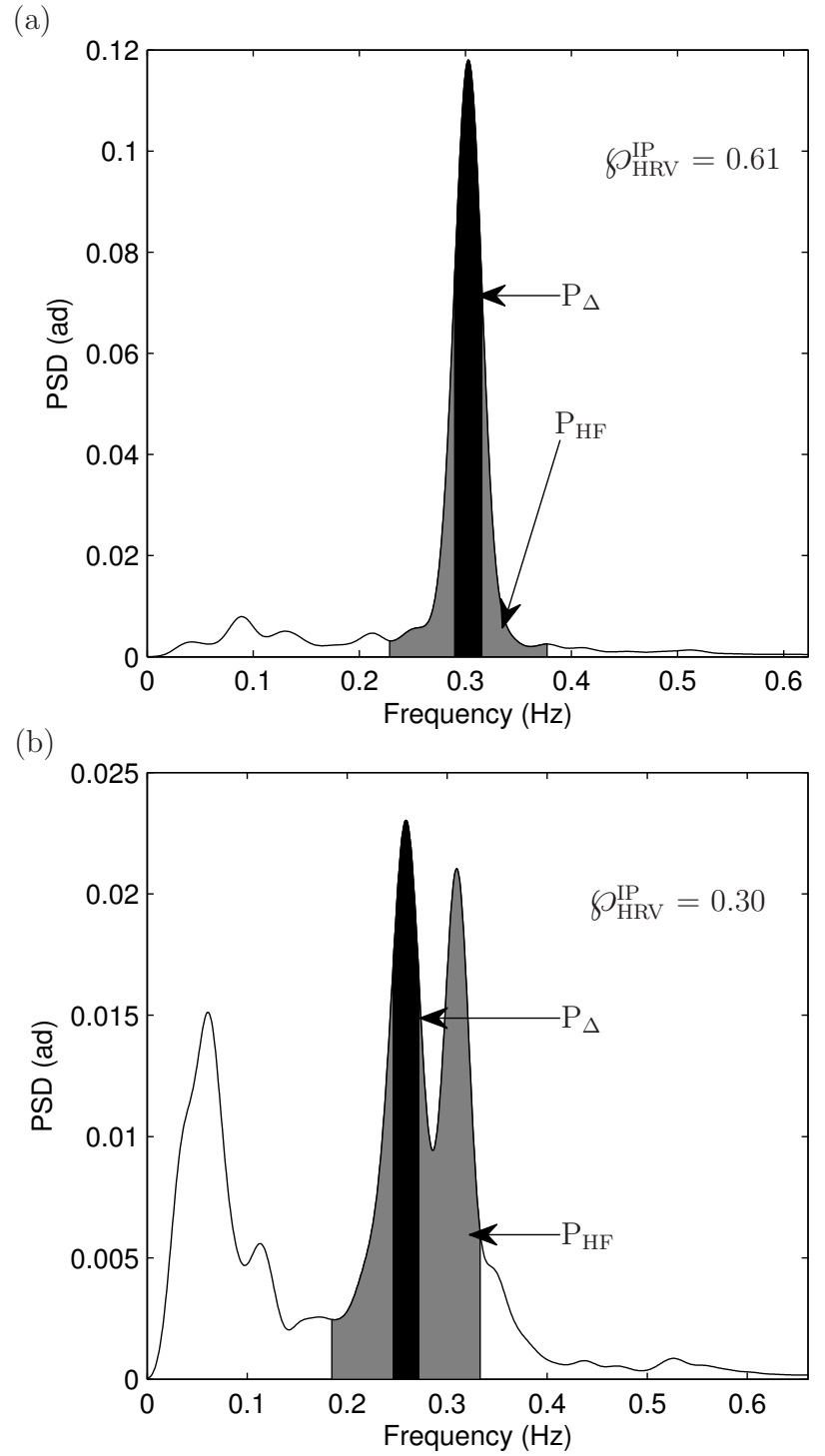

Fig. 3. $\wp_{\mathrm{HRV}}^{\mathrm{IP}}$ is obtained as the ratio between the area defined by the HRV spectrum between $\mathrm{F}_{\text {IP }}-\Delta f / 2$ and $\mathrm{F}_{\text {IP }}+\Delta f / 2$ (black, $\mathrm{P}_{\Delta}$ ) and the total area of the HF band (gray, $\mathrm{P}_{\mathrm{HF}}$ ). Note that whereas in (a) the existence of only one dominant frequency component results in a high $\wp_{\mathrm{HRV}}^{\mathrm{IP}}$, the presence of other powerful spectral components in HF band observed in (b) leads to a lower value. PSD, as obtained from the modulating signal $m(n)$ (see text for details), is adimensional (ad).

Comparing the results obtained for each of the groups, increased values of the three different $\wp$ and also decreased values of $R_{L F / H F}$ and $P_{L F n}$ were obtained for HiR and ICS with respect to LoR. The median values of the three different versions of $\wp$ are consistent within each group, being similar in the case of $\wp_{\mathrm{HRV}}^{\mathrm{IP}}$ and $\wp_{\mathrm{HRV}}^{\mathrm{EDR}}$ and slightly increased in the case of $\wp_{\mathrm{IP}}^{\mathrm{IP}}$. No noticeable differences could be assessed in the median values of the other considered parameters, although increased IQR was found in HiR for most of the classical time and frequency domain indexes.

As $\mathrm{P}_{\mathrm{HF}}$ mainly reflects parasympathetic activity and $\wp_{\mathrm{HRV}}^{\mathrm{IP}}$ aims to measure spectral distribution in HF band, it would be interesting to analyze if there exist a monotonic relationship between $\mathrm{P}_{\mathrm{HF}}$ and $\wp_{\mathrm{HRV}}^{\mathrm{IP}}$, as increased mean $\mathrm{P}_{\mathrm{HF}}$ could be related with increased $\wp_{\mathrm{HRV}}^{\mathrm{IP}}$. Spearman correlation 
coefficient was calculated between both parameters, being $\rho=0.38$, thus discarding monotony between them. On the other hand, possible relationship with sympathovagal balance was considered (lower $\mathrm{P}_{\mathrm{LFn}}$ was observed for HiR than for LoR), so correlation between $\mathrm{P}_{\mathrm{LFn}}$ and $\wp_{\mathrm{HRV}}^{\mathrm{IP}}$ was calculated. In this case, the result was $\rho=-0.72$ thus revealing a negative correlation between the indexes. This might indicate that higher values of $\wp_{\mathrm{HRV}}^{\mathrm{IP}}$ would be associated with parasympathetic dominance, as $\mathrm{P}_{\mathrm{LFn}}=\mathrm{P}_{\mathrm{LF}} /\left(\mathrm{P}_{\mathrm{LF}}+\mathrm{P}_{\mathrm{HF}}\right)$. Regarding $\wp_{\mathrm{HRV}}^{\mathrm{IP}}$ and $\wp_{\mathrm{IP}}^{\mathrm{IP}}, \rho=0.94$ was obtained suggesting strong correlation. However, Bland-Altman plot displayed in Fig. 4 suggests that both methods are not equivalent as range of confidence intervals is larger than the difference of the medians between groups (Table II). Negative bias indicates that $\wp_{\mathrm{IP}}^{\mathrm{IP}}$ usually present higher values (as can be also noticed in their median values displayed in Table II).

Finally, in Fig. 5, boxplots of mean $\wp_{\mathrm{HRV}}^{\mathrm{IP}}$ and $\mathrm{P}_{\mathrm{LFn}}$ for HiR and LoR groups are shown for each two-hour interval, in order to evaluate the robustness of each of the parameters to discriminate between both groups along the whole night. According to this figure, $\wp_{\mathrm{HRV}}^{\mathrm{IP}}$ is a much more robust index, as it is able to discriminate between both groups at almost every interval.

\section{DISCUSSION}

Several studies have pointed out to parasympathetic branch of ANS as the main responsible of broncho-constriction mechanisms [16], [17] and bronchomotor tone control [15], which are closely related to asthma. The increased vagal activity and altered autonomic airway control observed in asthmatic patients may be also reflected in cardiac vagal activity [13], [14] and hence HRV could be a suitable tool for evaluating those changes. In this work, we hypothesized that not only increased vagal tone but also a distinct behaviour of vagal activity could be related with asthma, and that these differences could be characterized through HRV spectral analysis. For this purpose, we defined $\wp$ as an index to evaluate the spectral distribution of HF components of HRV spectra, and we used it in the characterization of a group of preschool children that had been previously classified attending to their risk of developing asthma.

Concept of peakness, was already presented by Bailón et al. [27] and used by Lázaro et al. [31] and Hernando et al. [37] for respiratory frequency estimation and stress assessment, respectively. However, a modified definition is employed here. Essentially, the main differences between $\wp$ presented here and peakness proposed by Bailón et al. are related to the frequency resolution and to the frequency bands definition: whereas Bailón et al. aimed to distinguish those spectra with a clear dominant frequency, $\wp$ is used in this work for the characterization of the frequency distribution in the HF band. In this way, a higher resolution is employed here for calculating the PSD, as two near powerful frequency components must be differenced. Moreover, Bailón et al. considered a wider frequency band, as peakness was applied to respiratory signals which are not expected to show nonrespiration-related components. In difference, $\wp$ is applied

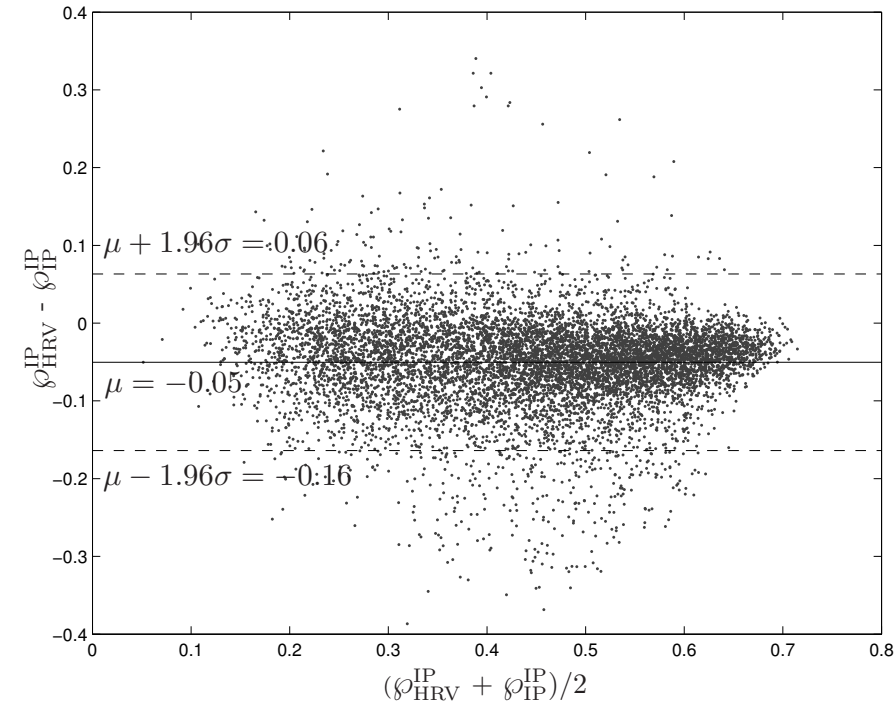

Fig. 4. Bland-Altman plot for agreement evaluation between $\wp_{\mathrm{HRV}}^{\mathrm{IP}}$ and $\wp_{\mathrm{IP}}^{\mathrm{IP}}$. In the figure, $\mu$ is the mean of $\wp_{\mathrm{HRV}}^{\mathrm{IP}}-\wp_{\mathrm{IP}}^{\mathrm{IP}}$, whereas $\sigma$ is the standard deviation of these differences.
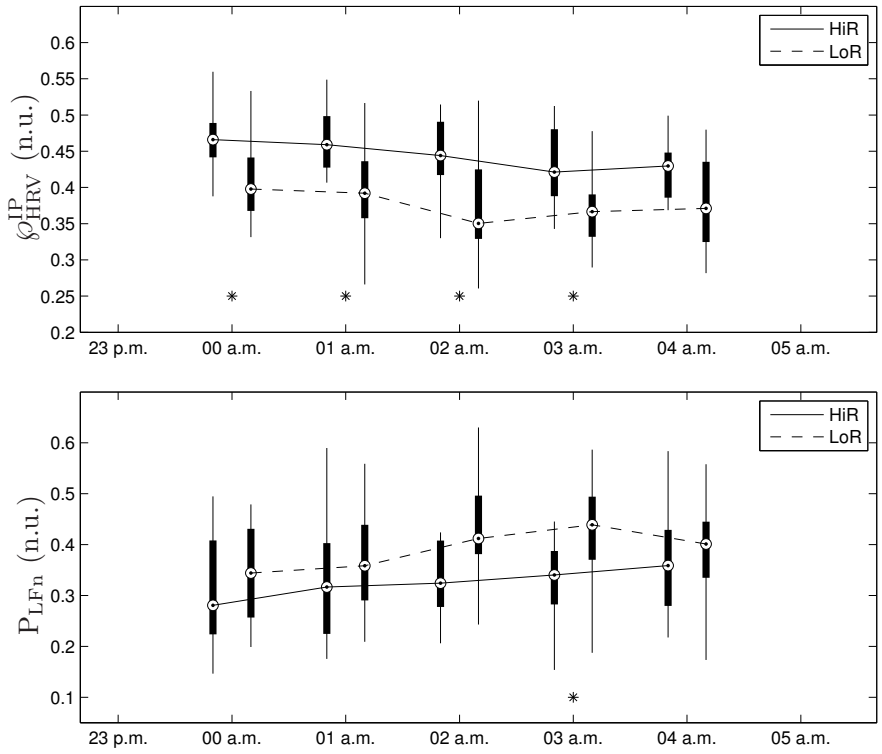

Fig. 5. Temporal evolution of the mean values of $\wp_{\mathrm{HRV}}^{\mathrm{IP}}$ and $\mathrm{P}_{\mathrm{LFn}}$ is shown. Boxplots are centered in the intermediate hour of the two-hour interval considered (boxplots for different groups, although plotted separately for interpretation purposes, are calculated with the same time references). * indicates significant differences between HiR and LoR in the given two-hour interval.

to HRV signals which are well known to have other nonrespiration-related modulations, thus a narrower band was considered.

$\wp_{\mathrm{HRV}}^{\mathrm{IP}}$ was analyzed together with classical time and frequency domain HRV indexes. Previously, HRV signals were conveniently preprocessed. Although it is well known that an accurate ectopics detection and correction is crucial in HRV analysis, the problem in this case is different: if we considered a RSA episode as ectopic (overcorrection), we would be loosing fundamental information, as RSA is essentially what we pretend to characterize through $\wp_{\mathrm{HRV}}^{\mathrm{IP}}$. RSA episodes 
TABLE II

MEAN VALUe BETWEen 02:00 AND 04:00 OF THE PRESENTED PARAMETERS FOR EACH OF THE GROUPS (MEDIAN (IQR)). * AND ** INDICATE DIFFERENCES WITH LOR $(p<0.05$ AND $p<0.005$ RESPECTIVELY $)$, WHILE $\dagger$ INDICATES DIFFERENCES WITH HIR $(p<0.05)$. AS $\mathrm{P}_{\text {LF }}, \mathrm{P}_{\text {HF }}$ AND TP ARE CALCULATED FROM $m(n)$ AND NOT DIRECTLY FROM RR INTERVAL SERIES, THEY ARE ADIMENSIONAL (AD).

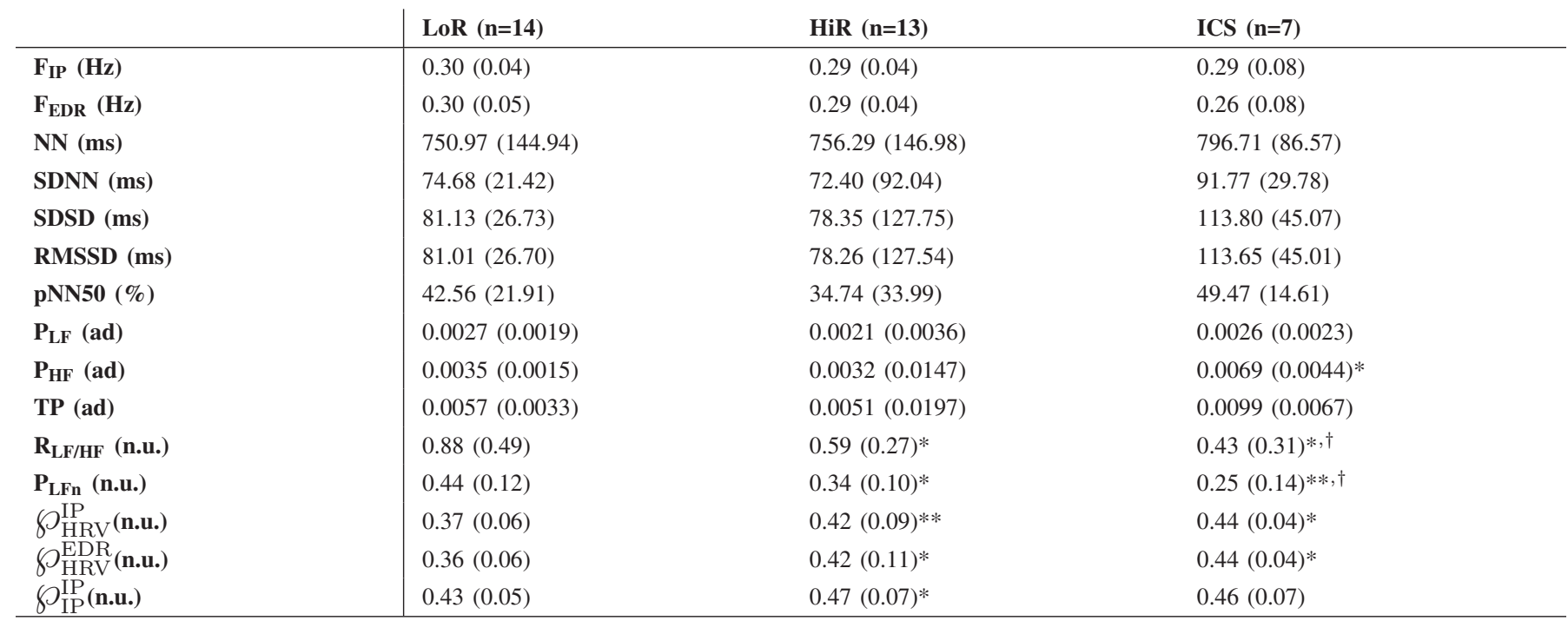

detector proposed here aims to minimize the number of false ectopic detections, which would lead to a smoother spectrum hence introducing a bias in the computation of $\wp_{\mathrm{HRV}}^{\mathrm{IP}}$. Also, the definition of the HF band was not the classical [0.15-0.4] $\mathrm{Hz}$ band recommended by the Task Force [18], instead it was defined as a $0.15 \mathrm{~Hz}$ band centered in the estimated respiratory rate, $F_{\text {IP }}$. As shown in Fig. 2, the lower frequency bound of the classical band could be inadequate when some not welldefined components (mid-frequency band [33]) are present, as power with an origin distinct from parasympathetic activity will contaminate the HF band. In addition, the upper bound of $0.4 \mathrm{~Hz}$ could be not enough when respiratory rate is high as some HF power will leak out of the band (young children present high respiratory rates [38]), so $\overline{\mathrm{HR}} / 2$ was considered as it is the highest representable frequency in HRV spectrum, due to the fact that HR is the intrinsic sampling frequency of HRV [34].

Results in Table II show statistical significant differences between LoR and HiR in $\wp_{\mathrm{HRV}}^{\mathrm{IP}}, \mathrm{R}_{\mathrm{LF} / \mathrm{HF}}$ and $\mathrm{P}_{\mathrm{LFn}}$, thus suggesting a peakier component in the HF band accompanied by a reduced sympathovagal balance in HiR. Also differences between LoR and ICS were assessed by the previously cited parameters, together with $\mathrm{P}_{\mathrm{HF}}$. Similar results obtained for HiR and ICS could reflect an intrinsic alteration of ANS behavior, although it may be difficult to infer whether this altered behavior could be caused by medication itself. Nevertheless, limited size of ICS group compromises the further physiological interpretation.

In a previous study, Emin et al. [14] reported increased PSNS activity and parasymapthetic dominance in older children (7-12 years) with a clinical diagnosis of asthma, as well as the ability of HRV analysis to stratify asthma severity. Here, increased parasympathetic dominance assessed in HiR and ICS by lower $R_{L F / H F}$ and $P_{L F n}$ is consistent with the results in
[14]. However, in difference with [14], mean $\mathrm{P}_{\mathrm{HF}}$ was similar in all the groups, which might be due to several reasons. First of all, distinct definitions of HF band are employed, as in [14] it is defined as $[0.15,0.5] \mathrm{Hz}$, thus impeding direct comparison of the results. Moreover, we are not performing a classification of children with diagnosed asthma but a characterization of groups that were formed attending to the predicted asthma risk, and also age differences between the populations of both studies may be accompanied by differences in the ANS functioning. Finally, the recordings in Emin et al. [14] were performed under predefined conditions of stimulated PSNS activity (deep breathing, Valsalva maneuver), whereas in our study ECGs were acquired without a controlled environment.

In a further step, a new definition of $\zeta$ without need of the IP signal was proposed. This new index, $\wp_{\mathrm{HRV}}^{\mathrm{EDR}}$, required estimation of the respiratory rate so an EDR signal was obtained from each ECG as proposed by Lázaro et al. [31]. Afterwards, F EDR was obtained as proposed by Bailón et al. [27]. Results from $\wp_{\mathrm{HRV}}^{\mathrm{EDR}}$ were similar to those obtained from $\wp_{\mathrm{HRV}}^{\mathrm{IP}}$ for the three groups and it was also able to distinguish HiR from LoR, thus supporting the use of only ECG signals and hence of a simpler acquisition hardware. However, this index requires $\mathrm{F}_{\mathrm{EDR}}$ estimation to be accurate. Here, a mean absolute error less than $0.005 \mathrm{~Hz}$ was achieved.

In order to verify whether $\wp_{\mathrm{HRV}}^{\mathrm{IP}}$ does reflect a measurement of the respiratory activity or either it reflects another mechanism, a third definition where only IP signal was considered, $\wp_{\text {IP }}^{\text {IP }}$, was proposed. Analogously to the previous definitions of $\wp$, higher $\wp_{\mathrm{IP}}^{\mathrm{IP}}$ was obtained for HiR and ICS and significant differences were found between LoR and HiR and hence it can be derived from the IP signal with no need of ECG. High correlation between both $\zeta$ definitions $(\rho=0.94)$ suggests strong relationship. However, Bland-Altman plot displayed in Fig. 4 suggests that both measurements are not equivalent, 
as range of confidence intervals is larger than the difference of the medians between groups (as can be noticed in Table II). In this way and despite the fact that both indexes are though to measure the same phenomenon, they cannot be used interchangeably, as the intrinsic existence of different spectral components in respiration and HRV lead to different measurements. As displayed in Fig. 4 and Table II, $\wp_{\text {IP presents }}^{\text {IP }}$ higher average values, thus overestimating the results obtained by $\rho_{\mathrm{HRV}}^{\mathrm{IP}}$.

Summarizing, the three definitions of $\varsigma$ lead to similar results, with increased values for HiR when compared with LoR. Hence, and as $\wp$ is a measurement of how the power is concentrated around the respiratory frequency, a peakier HF component seems to be a common feature of children with enhanced asthma risk. A possible explanation of increased $\wp_{\text {HRV }}^{\text {IP }}$ would be a strong correlation with $P_{H F}$. However, low Spearman correlation between both variables rejected this hypothesis. Nevertheless, negative correlation between $\wp_{\mathrm{HRV}}^{\mathrm{IP}}$ and $\mathrm{P}_{\text {LFn }}$ suggests that spectral distribution of $\mathrm{HF}$ components may be closely related with changes in sympathovagal balance, whose altered behavior has been proposed to be responsible of increased bronchoconstriction and bronchomotor tone observed in asthmatics [15]-[17]. In spite of that, the nature of these changes is not easy to analyze, as an increase in sympathetic nervous system activity often produces a similar effect than a decrease in PSNS activity and vice versa. Even though correlation between $\wp_{\mathrm{HRV}}^{\mathrm{IP}}$ and $\mathrm{P}_{\mathrm{LFn}}$ has been assessed, the former has been presented as a more robust index against inter- and intra-subject variability for distinguishing between HiR and LoR, as displayed in Fig. 5). In addition, $\wp$ appears to be robust, among young children, against the effect of sleep stages over HRV and respiratory frequency [39] which may be responsible of the lack of significant differences in $\mathrm{P}_{\mathrm{LFn}}$ between groups in most of the two-hour periods (see Fig. 5). As we do not dispose of polysomnographic recordings in this study, we cannot evalaute the effect of sleep stages over HRV or respiratory frequency. Nonetheless, additional confounders apart from risk of asthma could be considered: e.g., obstructive sleep apnea syndrome (OSAS) and chronic obstructive pulmonary disease (COPD) are also obstructive diseases causing dyspnea and that have been related with altered HRV [40], [41]. In the case of OSAS, visual analysis of the IP signals of the different patients have revealed the absence of generalized amplitude decreases that could indicate apneic episodes, and no differences between changes in IP amplitude were noticed in the different groups. To the authors knowledge, no COPD diagnosis was made for any of the subjects in the database, although it is true that COPD diagnosis is again compromised in so young children.

Despite being the physiological underlying processes difficult to interpret, increased $\zeta$ in HiR may be reflecting differences in the PSNS-driven control of respiratory modulation, since changes in respiratory pattern do affect RSA as suggested by Strauss-Blasche et al. [42]. In this way, HiR patients would be presenting a more stable respiration component, which could be related with a lack of adaptability of the whole respiratory system. This hypothesis is also supported by the results obtained by Seppä et al.
[22], who assessed reduced chaoticity in the IP of children with high-risk of developing asthma. Moreover, Veiga et al. assessed increased regularity of the airflow pattern in asthmatics through decreased approximate entropy [43]. In a previous study, we also observed a decreased complexity (measured through correlation dimension analysis) of the HF components in the case of HiR [44]. Hence, nonlinear analysis of the airflow and HRV in asthmatics (and potential asthmatics) suggests that asthma might be regarded as a state of increased regularity as hypothesized by Que et al. [19] or by Goldberger, who described illness as a state of reduced complexity [45]. Increased $\wp$ in children that are more likely to develop asthma in the future might be related with this 'decomplexification' and despite the hypothetical background, previous measurements suggesting decreased complexity in asthmatics could be reflecting a lack of adaptability. Hence, decreased adaptability of PSNS activity in early stages of life could be a potential marker of asthma risk. Nevertheless, in order to go deeper into the understanding of the nature of the differences between groups, a prospective analysis would be desirable so that we could assess if children classified as $\mathrm{HiR}$ are indeed developing asthma in the future, as the available classification is only based in the clinical history of the children, and without accounting for inflammatory markers. Additionally, the limited size of the database makes further study necessary to provide stronger evidence of the results reported here.

\section{CONCLUSION}

In conclusion, in this work HRV analysis has been used for the characterization of ANS activity in a group of preschool children divided in 3 groups based on the risk of asthma development and on medication intake: high-risk, low-risk, and under ICS treatment. Consistently with previous studies that described asthma as an state of increased regularity, peakness, $\wp$, has been presented as a robust index that reflects a peakier HF component in children with high risk of developing asthma in the future. As HRV analysis is non-invasive in nature, it may be regarded as a convenient tool to aid in the study of the neural mechanisms underlying asthma, thus complementing the information obtained through other methods.

\section{REFERENCES}

[1] W. Busse, H. Boushey, C. Camargo, D. Evans, M. Foggs, S. Janson, et al., "Expert panel report 3: Guidelines for the diagnosis and management of asthma," Washington, DC: US Department of Health and Human Services, National Heart Lung and Blood Institute, pp. 1-417, 2007.

[2] A. Sheffer, J. Bousquet, W. Busse, T. Clark, R. Dahl, D. Evans, L. Fabbri, F. Hargreave, S. Holgate, H. Magnussen, et al., "International consensus report on diagnosis and treatment of asthma.," Eur Resp $J$, vol. 5 , no. 5, pp. 601-641, 1992.

[3] F. Martinez, "Recognizing early asthma," Allergy, vol. 54, no. s49, pp. 24-28, 1999.

[4] P. F. Adams and M. A. Marano, "Current estimates from the national health interview survey, 1994.," Vital Health Stat 10, Data from the National Health Survey, no. 193 Pt 1, p. 1, 1995.

[5] C. M. Ionescu, "The human respiratory system," in The Human Respiratory System, pp. 13-22, Springer, 2013.

[6] J. Bousquet, P. K. Jeffery, W. W. Busse, M. Johnson, and A. M. Vignola, "Asthma: from bronchoconstriction to airways inflammation and remodeling," Am J Respir Crit Care Med, vol. 161, no. 5, pp. 1720$1745,2000$. 
[7] L. Agertoft and S. Pedersen, "Effects of long-term treatment with an inhaled corticosteroid on growth and pulmonary function in asthmatic children," Respir Med, vol. 88, no. 5, pp. 373-381, 1994.

[8] J. FitzGerald, E. Bateman, L. Boulet, A. Cruz, T. Haahtela, M. Levy, P. O. Byrne, P. Paggiaro, S. Pedersen, M. Soto-Quiroz, et al., "Global strategy for asthma management and prevention. 2015," Global Initiative for Asthma, 2015

[9] J. A. Castro-Rodríguez, C. J. Holberg, A. L. Wright, and F. D. Martinez, "A clinical index to define risk of asthma in young children with recurrent wheezing," Am J Respir Crit Care Med, vol. 162, no. 4, pp. 1403-1406, 2000.

[10] R. Kurukulaaratchy, S. Matthews, S. Holgate, and S. Arshad, "Predicting persistent disease among children who wheeze during early life," Eur Resp J, vol. 22, no. 5, pp. 767-771, 2003.

[11] D. Caudri, A. Wijga, C. M. A. Schipper, M. Hoekstra, D. S. Postma, G. H. Koppelman, B. Brunekreef, H. A. Smit, and J. C. de Jongste, "Predicting the long-term prognosis of children with symptoms suggestive of asthma at preschool age," J Allergy Clin Immunol, vol. 124, no. 5, pp. 903-910, 2009.

[12] M. Kaliner, J. H. Shelhamer, P. B. Davis, L. J. Smith, and J. C. Venter, "Autonomic nervous system abnormalities and allergy," Ann Intern Med, vol. 96 , no. 3, pp. 349-357, 1982.

[13] J. Kallenbach, T. Webster, R. Dowdeswell, S. Reinach, R. Millar, and S. Zwi, "Reflex heart rate control in asthma. evidence of parasympathetic overactivity.," Chest, vol. 87, no. 5, pp. 644-648, 1985.

[14] O. Emin, G. Esra, D. Aysegül, E. Ufuk, S. Ayhan, and D. M. Rusen, "Autonomic nervous system dysfunction and their relationship with disease severity in children with atopic asthma," Respir Physiol Neurobiol, vol. 183, no. 3, pp. 206-210, 2012.

[15] J. Morrison, S. Pearson, and H. Dean, "Parasympathetic nervous system in nocturnal asthma," Br Med J (Clin Res Ed), vol. 296, no. 6634, p. 1427,1988

[16] M. Lewis, A. Short, and K. Lewis, "Autonomic nervous system control of the cardiovascular and respiratory systems in asthma," Respir Med, vol. 100, no. 10, pp. 1688-1705, 2006.

[17] J. A. Nadel and P. J. Barnes, "Autonomic regulation of the airways," Anпи Rev Med, vol. 35, no. 1, pp. 451-467, 1984.

[18] Task Force of the European Society of Cardiology and others, "Heart rate variability standards of measurement, physiological interpretation, and clinical use," Eur Heart J, vol. 17, pp. 354-381, 1996.

[19] C.-L. Que, C. Kenyon, R. Olivenstein, P. T. Macklem, and G. N. Maksym, "Homeokinesis and short-term variability of human airway caliber," J Appl Physiol, vol. 91, no. 3, pp. 1131-1141, 2001.

[20] V.-P. Seppä, A. S. Pelkonen, A. Kotaniemi-Syrjänen, M. J. Mäkelä, J. Viik, and L. P. Malmberg, "Tidal breathing flow measurement in awake young children by using impedance pneumography," J Appl Physiol, vol. 115, no. 11, pp. 1725-1731, 2013.

[21] T. W. Guilbert, W. J. Morgan, R. S. Zeiger, L. B. Bacharier, S. J. Boehmer, M. Krawiec, G. Larsen, R. F. Lemanske, A. Liu, D. T. Mauger, et al., "Atopic characteristics of children with recurrent wheezing at high risk for the development of childhood asthma," J Allergy Clin Immunol, vol. 114 , no. 6, pp. 1282-1287, 2004.

[22] V.-P. Seppä, A. S. Pelkonen, A. Kotaniemi-Syrjänen, J. Viik, M. J. Mäkelä, and L. P. Malmberg, "Tidal flow variability measured by impedance pneumography relates to childhood asthma risk," Eur Resp $J$, vol. 47, no. 6, pp. 1687-1696, 2016.

[23] P. Busek, J. Vankova, J. Opavskỳ, J. Salinger, and S. Nevsimalova, "Spectral analysis of heart rate variability in sleep," Physiol Res, vol. 54, no. 4 , p. $369,2005$.

[24] J. Morrison and S. Pearson, "The effect of the circadian rhythm of vagal activity on bronchomotor tone in asthma.," Br J Clin Pharmacol, vol. 28, no. 5, pp. 545-549, 1989.

[25] M. Merri, D. C. Farden, J. G. Mottley, and E. L. Titlebaum, "Sampling frequency of the electrocardiogram for spectral analysis of the heart rate variability," IEEE Trans Biomed Eng, vol. 37, no. 1, pp. 99-106, 1990.

[26] J. P. Martínez, R. Almeida, S. Olmos, A. P. Rocha, and P. Laguna, "A wavelet-based ecg delineator: evaluation on standard databases," IEEE Trans Biomed Eng, vol. 51, no. 4, pp. 570-581, 2004.
[27] R. Bailón, L. Sörnmo, and P. Laguna, "A robust method for ecg-based estimation of the respiratory frequency during stress testing," IEEE Trans Biomed Eng, vol. 53, no. 7, pp. 1273-1285, 2006.

[28] J. Mateo and P. Laguna, "Analysis of heart rate variability in the presence of ectopic beats using the heart timing signal," IEEE Trans Biomed Eng, vol. 50, no. 3, pp. 334-343, 2003.

[29] W. Hrushesky, D. Fader, O. Schmitt, and V. Gilbertsen, "The respiratory sinus arrhythmia: a measure of cardiac age," Science, vol. 224, no. 4652, pp. 1001-1004, 1984.

[30] M. G. Tsipouras, D. I. Fotiadis, and D. Sideris, "An arrhythmia classification system based on the rr-interval signal," Artif Intell Med, vol. 33, no. 3, pp. 237-250, 2005.

[31] J. Lázaro, A. Alcaine, D. Romero, E. Gil, P. Laguna, E. Pueyo, and R. Bailón, "Electrocardiogram derived respiratory rate from qrs slopes and r-wave angle," Ann Biomed Eng, vol. 42, no. 10, pp. 2072-2083, 2014.

[32] R. Bailón, G. Laouini, C. Grao, M. Orini, P. Laguna, and O. Meste, "The integral pulse frequency modulation model with time-varying threshold: application to heart rate variability analysis during exercise stress testing," IEEE Trans Biomed Eng, vol. 58, no. 3, pp. 642-652, 2011.

[33] Y. Goren, L. R. Davrath, I. Pinhas, E. Toledo, and S. Akselrod, "Individual time-dependent spectral boundaries for improved accuracy in time-frequency analysis of heart rate variability," IEEE Trans Biomed Eng, vol. 53, no. 1, pp. 35-42, 2006.

[34] J. Bolea, E. Pueyo, M. Orini, and R. Bailón, "Influence of heart rate in nonlinear hrv indices as a sampling rate effect evaluated on supine and standing," Front Physiol, vol. 7, p. 501, 2016.

[35] I. Feinberg, "Changes in sleep cycle patterns with age," J Psychiatr Res, vol. 10 , no. 3 , pp. $283-306,1974$.

[36] D. G. Altman and J. M. Bland, "Measurement in medicine: the analysis of method comparison studies," The Statistician, pp. 307-317, 1983.

[37] A. Hernando, J. Lázaro, E. Gil, A. A. Valdes, J. Garzon-Rey, R. LópezAntón, C. de la Cámara, P. Laguna, J. Aguiló, and R. Bailón, "Inclusion of respiratory frequency information in heart rate variability analysis for stress assessment," IEEE J Biomed Health Inform, vol. 20, no. 4, pp. 1016-1025, 2016.

[38] S. Fleming, M. Thompson, R. Stevens, C. Heneghan, A. Plüddemann, I. Maconochie, L. Tarassenko, and D. Mant, "Normal ranges of heart rate and respiratory rate in children from birth to 18 years of age: a systematic review of observational studies," The Lancet, vol. 377, no. 9770, pp. 1011-1018, 2011.

[39] R. Cabiddu, S. Cerutti, G. Viardot, S. Werner, and A. M. Bianchi, "Modulation of the sympatho-vagal balance during sleep: frequency domain study of heart rate variability and respiration," Front Physiol, vol. 3, 2012 .

[40] C. Varon, A. Caicedo, D. Testelmans, B. Buyse, and S. Van Huffel, "A novel algorithm for the automatic detection of sleep apnea from singlelead ecg," IEEE Trans Biomed Eng, vol. 62, no. 9, pp. 2269-2278, 2015.

[41] N. D. Giardino, L. Chan, and S. Borson, "Combined heart rate variability and pulse oximetry biofeedback for chronic obstructive pulmonary disease: preliminary findings," Appl Psychophysiol Biofeedback, vol. 29, no. 2, pp. 121-133, 2004.

[42] G. Strauss-Blasche, M. Moser, M. Voica, D. McLeod, N. Klammer, and W. Marktl, "Relative timing of inspiration and expiration affects respiratory sinus arrhythmia," Clin Exp Pharmacol Physiol, vol. 27, no. 8, pp. 601-606, 2000.

[43] J. Veiga, R. C. Faria, G. P. Esteves, A. J. Lopes, J. M. Jansen, and P. L. Melo, "Approximate entropy as a measure of the airflow pattern complexity in asthma," in $E M B C, 2010$ Annual International Conference of the IEEE, pp. 2463-2466, IEEE, 2010.

[44] J. Milagro, E. Gil, J. Bolea, V.-P. Seppä, L. P. Malmberg, A. S. Pelkonen, A. Kotaniemi-Syrjänen, M. J. Mäkelä, J. Viik, and R. Bailón, "Nonlinear dynamics of heart rate variability in children with asthmatic symptoms," in EMBEC \& NBC 2017, pp. 815-818, Springer, 2017.

[45] A. L. Goldberger, "Fractal variability versus pathologic periodicity: complexity loss and stereotypy in disease," Perspect Biol Med, vol. 40, no. 4, pp. 543-561, 1997. 